\title{
A Passive Positioning and Supporting Device for Surgical Robots and Instrumentation
}

\author{
A. Grey Lerner, M.S. ${ }^{2}$, Dan Stoianovici, Ph.D. ${ }^{1,2}$, Louis L. Whitcomb, Ph.D. ${ }^{2}$, \\ and Louis R. Kavoussi, M.D. ${ }^{1}$ \\ 1 James Buchanan Brady Urological Institute \\ Johns Hopkins Medical Institutions, Johns Hopkins University, Baltimore, Maryland, \\ USA \\ 2 Department of Mechanical Engineering \\ Whiting School of Engineering, Johns Hopkins University, Baltimore, Maryland, USA
}

\begin{abstract}
The recent development of compact surgical robots and instrumentation raise the need of a suitable mechanism for positioning and support in the proximity of the operative field. The accuracy of the surgical instrument and surgical procedure heavily relies on the ability of the supporting device to provide a sturdy base under the payload and dynamics of the instrument.

We present a compact and sturdy passive mechanical arm equipped with a central braking system that can be easily manipulated to desired locations and firmly locked in place. The arm presents serial link architecture comprising two links and three joints: spherical-rotational-spherical. To ease the maneuverability of the arm, one degree of freedom of the spherical joint at the base has been blocked, yielding six overall degrees of freedom.

The novelty of the arm relies in the special design of the braking mechanism that simultaneously locks all the joints using one single electric motor. The arm design is simple yet safe. One of its safety features is the power-fail-safe design rendered by the normally locked braking mechanism unlocked by a low-voltage electrical actuator.

The arm is designed for stand-alone use in the operating room as an independent module, representing the latest member of a growing family of surgical robotic modules under development at our institution.
\end{abstract}

\section{Introduction}

A significant research effort is presently dedicated to the development of purposebuilt surgical robotic systems. As compared to industrial types, these robots present distinctive features such as safety, sterility, and compactness making them appropriate for surgical environments. The reduced size, compact geometry, and lightweight of the mechanical structure are important robotic requirements necessary for satisfying ergonomic requirements in the operating room [2], [4]. Moreover, for image guided procedures the robot design must allow access within the limited working space of existing medical imagers [5], [6]. 
A shortcoming of the compact design is the reduced working envelope of the robot [7]. Although sufficiently large for performing the surgical procedure at hand, the working envelope is too narrow for providing global positioning over the patient from a base to the operated organ. A positioning and supporting device is thus required in order to initially locate the robot at the operative site and to sustain it during the procedure. The accuracy of the robot/instrument and the clinical outcome of the procedure heavily rely on the ability of the supporting arm to provide a fix reference base for the operation under the payload and dynamics of the robot/instrument. Thus such arms are required to be lightweight, compact, sturdy, and easy to position.

Several arms have been developed for supporting instrumentation on the operating table. They range from simple passive knob-locked clamps for organ retraction and instrument holding to active robotic holders providing actuated orientation of laparoscopic instruments (AESOP - Automated Endoscope and System for Optimal Positioning, Computer Motion, Inc., Goleta, CA). Several of these are equipped with joint position sensors [5]. The locking mechanisms employed are either individual for each joint or central, providing simultaneous locking of all joints. The common actuation used for the locking mechanism is manual [1] yet pneumatic (Leonard Arm, Leonard Medical, Inc., Huntingdon Valley, PA) and piezoelectric actuation [2] have been proposed. Several devices are either in the experimental stages of development while other are already commercially available, approved by regulatory committees, and clinically used (AESOP, Leonard). Generally, the existing systems well perform the particular task that they have been designed for. Nevertheless, in demanding applications such as the support of compact surgical robots and precision instrumentation, these arms present the common shortcoming of insufficient stiffness and payload capability.

This paper reports the design and performance evaluation of an arm capable to provide a steadier support of larger payloads, the Grey Arm. The compact and ergonomic characteristics of previous designs have been maintained while improving overall safety and maneuverability. The design is protected by a provisional patent of invention [3].

\section{Methods}

The main design requirements for the Grey Arm were specified according to the shortcomings of existing designs: increased stiffness and payload capability. For ergonomic and ease of use reasons, the arm was required to provide free, frictionless motion of the end-effector in the unlocked state. The locking mechanism requirement was to simultaneously engage all joints from one simple command. For the safety of operation in the event of power failure, the locking mechanism was required to present a normally locked configuration. Detailed specifications are given in Table 1. The SRS (spherical - revolute - spherical) kinematic architecture of the arm has been imposed as a design requirement due to the positive experience of previous SRS arms for providing a large working envelope in a 
Table 1. Design specifications

\begin{tabular}{ll}
\hline CONSIDERATION & SPECIFICATION \\
\hline Weight & $\leq 10 \mathrm{Kg}$ for comfortable single person operation \\
Degrees of freedom & $\geq 6$ preferable 6 \\
Workspace & Spherical with $0.35 \mathrm{~m}$ radius \\
End-effector & Easy attachment \\
Architecture & Spherical - Revolute - Spherical joints (SRS) \\
Payload & $\geq 10 \mathrm{Kg}$ \\
Actuation & Passive \\
Brakes & Simultaneous for all joints, \\
& normally locked, electrical actuation preferred \\
Locking time & $\leq 250 \mathrm{~ms}$ \\
Power & Electrical $\leq 24 \mathrm{~V}$ \\
Rigidity & $\geq 10 \mathrm{~Hz}$ natural frequency \\
Stiffness & $\geq 0.4 \mathrm{~N} / \mu \mathrm{m}(\sim 250 \mu \mathrm{m}$ deflection for $10 \mathrm{Kg}$ load at end effector $)$ \\
Materials & Non-corrosive \\
\hline
\end{tabular}

compact design. In addition, initial specifications required the arm to be passive in order to achieve simplicity and cost efficiency. Electrical actuation of the braking mechanism was preferred for its reliable, noiseless, and clean operation. Safety required a low voltage electrical supply.

Figure 1 presents a schematic of the Grey Arm. The arm comprises a proximal link (1) and a distal link (2) connecting three joints $(3,4,5)$. The middle joint (4) is revolute while the base joint (4) and the distal joint (5) are spherical.

The base of the arm is mounted via a tapered shaft (6) to a custom clamp (not represented) that mounts on a fixed support such as the rail of the operating room table. The surgical instrument or robotic system is connected as an endeffector at the distal joint (5) through a custom-made adapter (8). Both the base clamp and the end-effector adapter are simple and easy to manufacture. They can be machined depending on the desired base and end-effector of the given application.

\subsection{Degrees of Freedom}

The arm may be configured so that when unlocked it exhibits either seven or six overall degrees of freedom (dof). This wrench-adjustable configuration may be easily set depending on the application at hand and the preference of the surgeon.

The freedom of the arm results from the type and number of its joints. As previously described, the Grey Arm presents a spherical-cylindrical-spherical joint architecture altogether yielding seven $(3+1+3)$ degrees of freedom. In this situation the arm presents one redundant degree of freedom creating ambiguity in the orientation of the arm for a given end-effector location. This is, with more than 


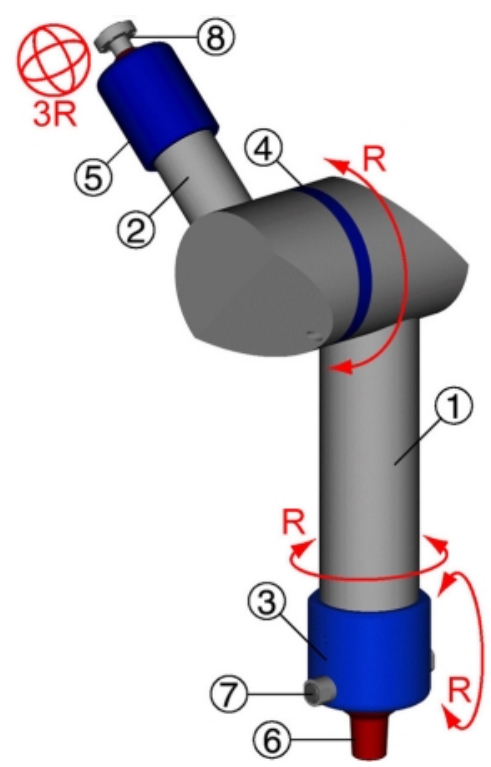

Fig. 1. Grey Arm: Architecture and degrees of freedom

six dof the linkage position is not unique. For the surgeon positioning the arm this translates into the requirement of holding not just the end-effector but also the arm, which sometimes may be impractical. However, the 7-dof configuration of the arm is necessary in case that specific orientations of the arm are desired.

Constraining the spherical (ball) joint at the base to only two rotational dof leads to less ambiguity in the positioning task. Ball joints are compact, simple, and free to rotate in three dimensions. By contrast, common 2-dof rotary joints, which are implemented by serially linking a pair of revolute joints, lead to a bulkier more complex assembly. A novel design was used to implement a ball joint with only two rotational dof (Figure 2).

The 2-dof ball joint employs a spherical ball (1) with an equatorial groove of semi-circular cross section (2) and a pair of spring loaded ball plungers (3) mounted into the socket of the joint (4). The plungers face opposite sides of the sphere (1) such that their spring loaded balls (5) ride smoothly in the mating equatorial groove (2). In this arrangement, one rotational dof is constrained while rotations $R_{\alpha}$ and $R_{\beta}$ are free. This constrain is active as long as the moment applied in the constrained direction is low. After a certain limit, which is determined by the plunger spring, the balls (5) leave the equatorial groove (2) and the blocked dof is released. This threshold is set so that under normal operation the joint is constrained, while under intentional action of the surgeon the constrain is bypassed allowing the arm to change orientation. In case that this operation mode is impractical for a certain clinical procedure the plungers (3) 


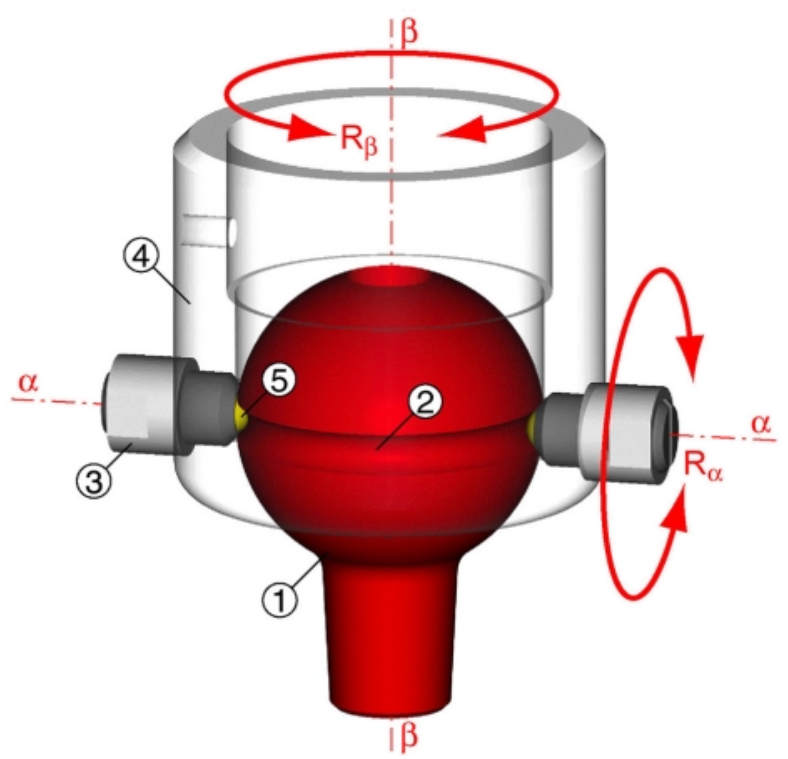

Fig. 2. The two degrees of freedom ball joint at the base of the Grey Arm

may be unloaded or completely removed thus rendering a regular 3-dof spherical joint.

This scheme allows the construction of a 2(3)-dof rotary joint while maintaining the compactness and simplicity of the classic spherical joint. In addition, the ball-based scheme allows the addition of a simple locking mechanism of the joint, as presented next.

\subsection{Central Active Locking Mechanism}

All joints of the arm are locked from one single electrical motor through a special mechanism that generates and transmits the locking force independent of the orientation of the arm. Figure 3 presents a planar axial cross section through the arm when the joints are oriented such that their axes are contained into the plane of the section. The links represented by the hallow tubes (4) and (17) are rigidly fixed into the ball sockets (3) respectively (19) and into the hubs of the middle joint (13) respectively (16). The electrical motor (6) used for the prototype is a $21.2 \mathrm{~W}$ power $12 \mathrm{~V}$ nominal voltage motor with a 159:1 planetary gear head (MicroMo Electronics: 3042W012CR+30/1 159:1). The motor is mounted into the housing (5) that is constrained to rotate into the link (4). The motor engages a planar cam mechanism represented by the thrust bearing (7), the cam (8), the pair of spherical balls (9), the balls race (10), and the cam follower (11). Figure 4 presents an isometric view of the cam (8), balls (9), and follower (11) represented transparent. Part numbers coincide with those in Figure 3. For clarity, in this representation the race of the ball race (10) has been hidden. 


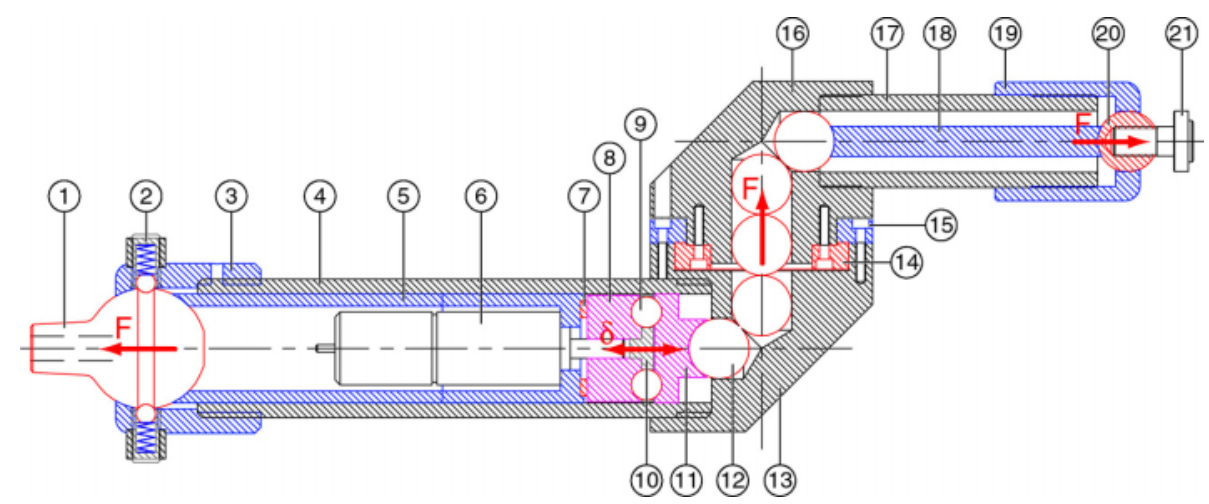

Fig. 3. Axial cross section of the Grey Arm

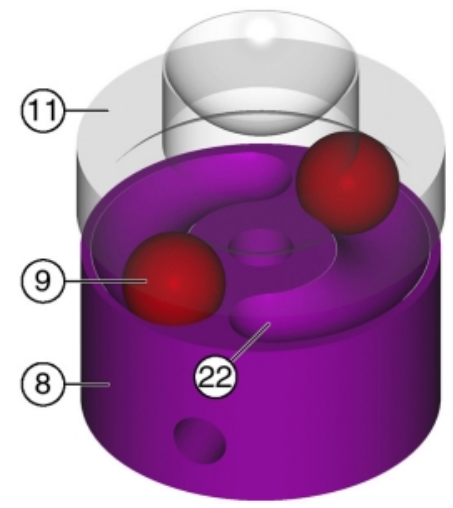

Fig. 4. The planar cam mechanism with load-carrying ball bearings

The cam (8) presents a pair of helical grooves of semi-circular cross section (22), end to end spanning $1.5 \mathrm{~mm}$ in height over $130^{\circ}$ each. The depth of the helical groove is a quadratic function of the helical angle, such that the mechanism provides large displacement at the beginning and large force at the end of the locking stroke. The race of balls (10) presents a small ball plunger (not represented) oriented radial which is in permanent contact with the inner part of the link (4). This is necessary in order to insure the engagement of the balls (9) between the cam (8) and the follower (11) independent of the arm's orientation.

The cam mechanism generates an axial displacement $\delta$ (Figure 3). A transmission mechanism comprising a series of spherical balls (12) and the spacer rod (18) propagates the displacement from the proximal joint (1) through the middle joint $(14,15)$ to the distal joint $(20)$. The chain of internal components is constrained within the outer components of the arm. Braking occurs when the length of the transmission chain $(1,5,8,9,11,18,20)$, which is controlled by the electrical motor $(\delta)$, exceeds the length of the conformed space $(3,4,13,16,17,19)$. 
This is guaranteed by the setting the cam displacement to be greater than the free travel in the transmission. An initial adjustment is used to fine tune the outer chain length by tightening the socket (19) on the distal link (17) such that in the unlocked state there is no braking force generated into the joins.

The cam displacement $\delta$ generates compressive forces throughout the entire internal path balanced by tensile forces in all outer components. This yields equal braking forces $F$ in all joints regardless of the relative orientation of the distal and proximal links. A reversed arrangement for the middle joint comprising the braking disks $(14,15)$ was necessary in order to achieve braking the under tensile forces. The disks $(14,15)$ are pushed together when the normal force $F$ attempts to spread the central joint, thus causing friction forces that block the joint.

\subsection{The Electronic Circuitry}

The Grey Arm is a modular component in that it functions independently only requiring a low voltage DC power supply. All components, including the electronic circuitry for motor control and the command buttons, are integrated into the arm. A power connector is mounted into the proximal sphere (1). The power is transmitted through a rotary connector to the electronic board located inside the proximal link between the ball (1) and the motor (6).

The command is based on two momentary buttons mounted on opposite sides of the proximal link. The arm unlocks when the buttons are simultaneously pressed. The arm locks as soon as at least one button is released. While the buttons are pressed the motor spins continuously in the unlocking direction. When a button is released the motor reverses for a short period that is timed to provide full locking of the arm. A current amplifier is used to limit the motor current. No encoders are required for operation.

\section{Results}

Two prototypes of the Grey Arm have been developed. The second generation improved upon esthetics and modularity. Figure 5 presents a photograph of the second prototype Grey Arm (1) supporting the driver (2) PAKY [6] of the trocar needle (3) under an x-ray fluoroscope (4) (C-Arm). The arm (1) is mounted by means of a clamp (5) on a rigid custom side rail (6) of the operating room table. The operation of the Grey Arm is simple and intuitive. The arm is normally locked and two buttons (7) must be pressed before the arm will unlock. While holding both buttons closed, the arm's 6/7-dof are fully available for completing the positioning task. Upon release of either button, the arm locks rigidly in place (approx. 200 ms locking time), fixing the position and orientation of the end-effector mounted at the distal joint.

Tests have been performed to assess performance and reliability. Experiments reviled that the Grey Arm prototype is concurrent with the design specifications (Table 2). 


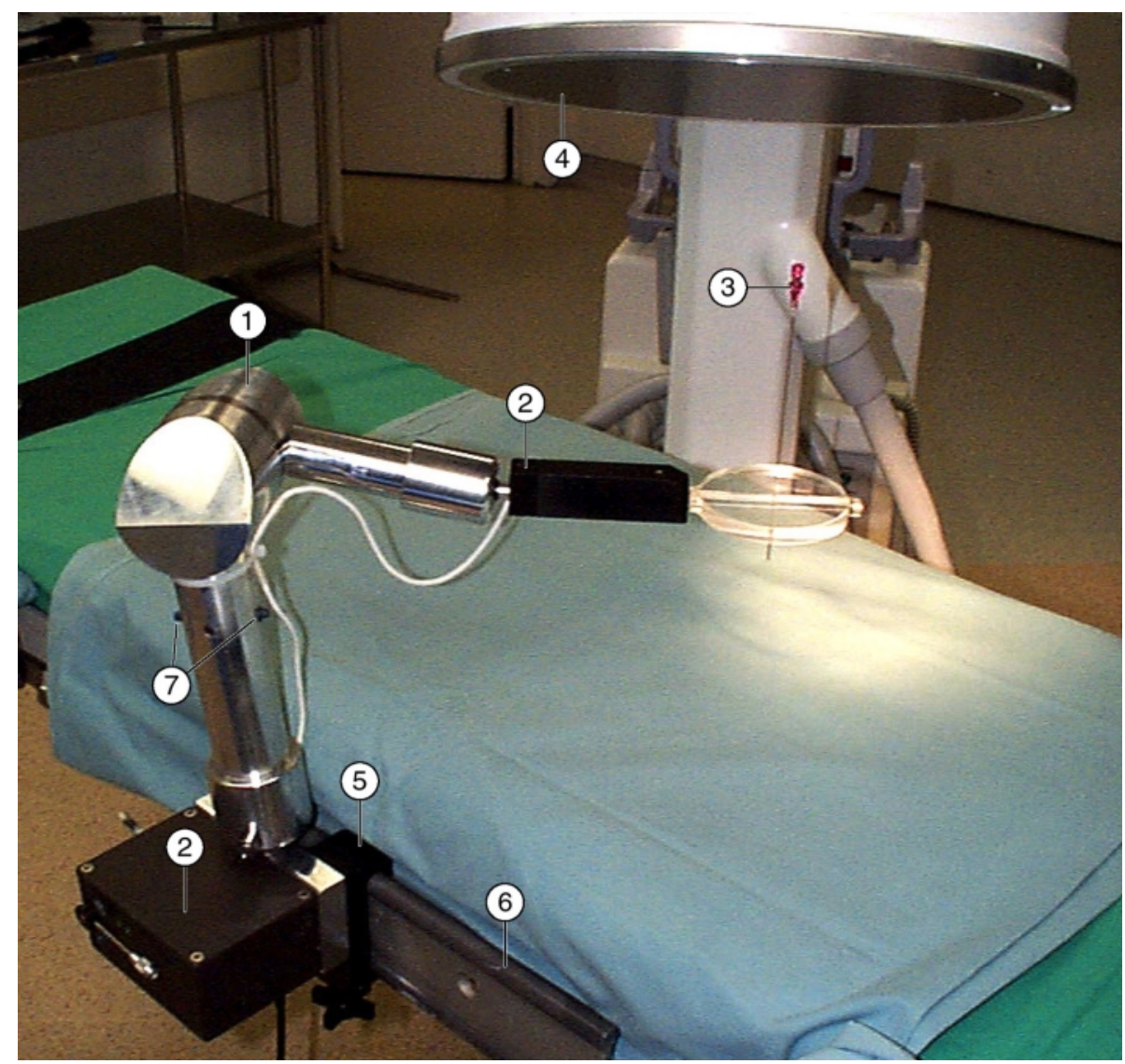

Fig. 5. The Grey Arm supporting the PAKY needle driver

High stiffness of the arm was imperative due to the nature of the task. Figure 6 presents the results of stiffness measurement experiments performed on the Grey Arm. The graph of the displacement versus load at the end-effector reveals sub-millimeter deflections realized at the end of the arm under full load.

Additional tests have been performed to assess the arm's rigidity by performing measurements of the natural frequency of the arm. An accelerometer interfaced with a digital spectrum analyzer was used to determine the natural vibration modes on the Grey Arm. The arm was found to be very rigid with a natural frequency of approximately $45 \mathrm{~Hz}$.

The Grey arm significantly increased upon the payload capability, rigidity, and safety of previous designs. For example, the piezoelectric arms developed at the Aachen University of Technology, Germany [2] exhibit 35N - 50N payload capability and 100 ms lock time using 200V - 1000V electrical power. Our design 
Table 2. Achieved and specified design considerations

\begin{tabular}{lll}
\hline CONSIDERATION & SPECIFICATION & PROTOTYPE \\
\hline Weight & $\leq 10 \mathrm{Kg}$ & $6.65 \mathrm{Kg}$ \\
Degrees of freedom & $\geq 6$ preferable 6 & $6 \mathrm{or} 7$ \\
Workspace & Spherical with 0.35 m radius & $0.4 \mathrm{~m}$ \\
End-effector & Easy attachment & $\mathrm{M} 12 \mathrm{x} 1.5$ thread \\
Architecture & Spherical - Revolute - Spherical joints (SRS) & $\mathrm{SRS}$ \\
Payload & $\geq 10 \mathrm{Kg}$ & $19 \mathrm{Kg}$ \\
Actuation & Passive & Yes \\
Brakes & Simultaneous, normally locked, electrical & Yes \\
Locking time & $\leq 250 \mathrm{~ms}$ & $200 \mathrm{~ms}$ \\
Power & Electrical $\leq 24 \mathrm{~V}$ & $24 \mathrm{~V}$ \\
Rigidity & $\geq 10 \mathrm{~Hz}$ natural frequency & $45 \mathrm{~Hz}$ \\
Stiffness & $\geq 0.4 \mathrm{~N} / \mu \mathrm{m}$ & $0.7 \mathrm{~N} / \mu \mathrm{m}$ \\
& $(\sim 250 \mu \mathrm{m}$ @ $10 \mathrm{Kg})$ & $(137 \mu \mathrm{m})$ \\
Materials & Non-corrosive & Aluminum, \\
& & stainless steel, \\
& & teflon \\
\hline
\end{tabular}

revealed a four times increase of payload ( $200 \mathrm{~N}$ ), uses a safe voltage supply of $24 \mathrm{~V}$ DC, while slightly increasing the braking time to $200 \mathrm{~ms}$.

During testing, the Grey Arm did not malfunction and proved to be reliable. The Grey Arm presents several features that insure safe operation. The arm is normally locked, hence power failures do not lead to arm unlocking. In addition, dual buttons insure that unlocking does not occur inadvertently. The arm operates on a low power supply, which is safe for the patient as well as the medical personnel. While firmly fixed in the locked position, the arm could forcibly be moved should a situation necessitate such action.

Clinical experiments with the Grey Arm supporting the RCM-PAKY system [7] will soon commence. The system will be initially used for performing image guided percutaneous renal access in the operating room using a portable $\mathrm{x}$-ray imager.

\section{Conclusion}

A fully functional Grey Arm prototype has been designed and manufactured. Testing of the device shows that it meets or exceeds all given design specifications. Clinical trials will begin in the near future.

The design includes two new features: the architecture of the central locking mechanism and the design of the 2-dof spherical joint. With these features, the compact-ergonomic characteristic of previous designs has been maintained while improving overall safety, reliability, maneuverability, rigidity, and payload capability. The arm is intuitive and simple to use. 


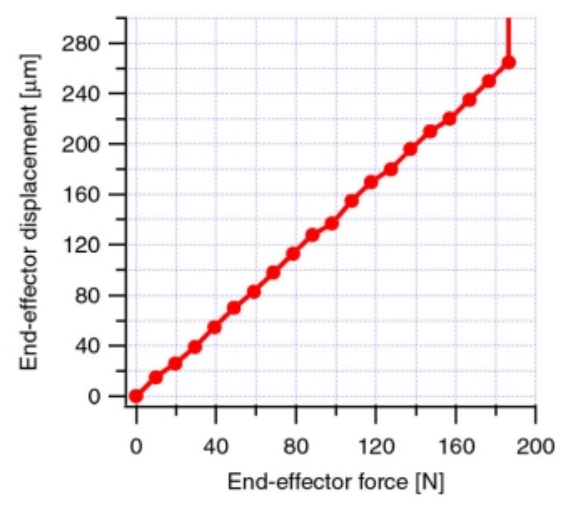

Fig. 6. Stiffness diagram for the Grey Arm

The arm shares the attributes of compactness and modularity of the robotic systems / surgical instruments for which will provide support on the operating table. This stand-alone device requires no additional components and operates on a DC power supply.

This type of device may prove to be highly useful for holding, supporting, and stabilizing a great variety of robotic and conventional surgical devices. We are hoping to begin clinical use of this device in the near future.

\section{Acknowledgements}

We thank Mr. Leonard Bonell of Leonard Medical Inc., Huntingdon Valley, PA for partially supporting the research involved in the development of the prototype. This research was also supported in part by the National Science Foundation under grants BES-9625143 and EEC-9731748.

\section{References}

1. J. A. Cadeddu, D. Stoianovici, L. R. Kavoussi. Robotics in urologic surgery. Urology, 49(4):501-507, 1997. 1053

2. S. Erbse, K. Radermacher, M. Anton, G. Rau, W. Boeckmann, G. Jakse, H. W. Staudte. Development of an Automatic Surgical Holding System Based on Ergonomic Analysis. 1997 CVRMed - MrCas, Lecture Notes in Computer Science, Springer-Verlag, 1205:737-746, 1997. 1052, 1053, 1059

3. G. A. Lerner, D. Stoianovici, L. L. Whitcomb, L. R. Kavoussi. A Passive Positioning Device for Surgical Robots and Instrumentation. Provisional patent of invention, DM-3489, 1998. 1053

4. P. Potamianos, B. L. Davies, R. D. Hibberd. Intra-operative imaging guidance for keyhole surgery methodology and calibration. Proc. First Int. Symposium on Medical Robotics and Computer Assisted Surgery, Pittsburgh, PA., 98-104, 1994. 1052 
5. P. Potamianos, B. L. Davies, R. D. Hibberd. Intra-operative registration for percutaneous surgery. Proc. Second Int. Symposium on Medical Robotics and Computer Assisted Surgery, Baltimore, MD., 156-164, 1995. 1052, 1053

6. D. Stoianovici, J. A. Cadeddu, R. D. Demaree, H. A. Basile, R. H. Taylor, L. L. Whitcomb, W. N. Sharpe Jr., L. R. Kavoussi. An Efficient Needle Injection Technique and Radiological Guidance Method for Percutaneous Procedures. 1997 CVRMed - MrCas, Lecture Notes in Computer Science, Springer-Verlag, 1205:295298, 1997. 1052, 1058

7. D. Stoianovici, L. L. Whitcomb, J. H. Anderson, R. H. Taylor, L. R. Kavoussi. A Modular Surgical Robotic System for Image Guided Percutaneous Procedures. 1998 MICCAI, Lecture Notes in Computer Science, Springer-Verlag, 1496:404-410, 1998. 1053, 1060 